the new proposals there would be 321 initial referrals, a $65.7 \%$ reduction. If all of these underwent colonoscopy calculated cost would be $£ 218 \mathrm{k} .279$ patients fell into the repeat testing category (costing $£ 8 \mathrm{k}$ ). Introducing new guidelines would predict a saving of $£ 43 \mathrm{~K}$ on colonoscopy at a laboratory expense of $£ 8 \mathrm{k}$.

Conclusions Implementation of the potential new primary care guidelines could result in markedly fewer referrals to secondary, with a small increase in laboratory workload, and could thus reduce the strain for secondary care services. In SW London this could save $£ 35 \mathrm{k}$, and lead to a reduction in secondary care referrals but lead to an increase in primary care reviews.

\section{PTU-139 THE NEED TO WORK 'SMART' ON ACUTE WARDS: COULD MOBILE PHONE APPLICATIONS BE THE ANSWER?}

Haroon Khan*, Richard Warner, James Ritchie, Kush Sharma, Andrew Robinson, Marie McMahon, Dipesh H Vasant. Salford Royal Foundation Trust, Salford, UK

\subsection{6/gutjinl-2018-BSGAbstracts.517}

Introduction Due to rising service pressures there is a need for innovation to optimise efficiency on acute medical and gastroenterology wards. Using smartphone applications to achieve this is an attractive proposition, but the utility of such technologies in clinical practice is unclear. Here, we report the experience of multidisciplinary staff after a pilot of a smartphone application at our hospital.

Methods Two acute medical wards (including a 24 bed gastroenterology ward), were selected for a two-week pilot trial of a smartphone application, Listrunner (Desma Health, Canada), during October 2017. Multidisciplinary team members including; doctors, pharmacists, and nurses, were given access to Listrunner via dedicated secure mobile devices. During daily consultant ward rounds and throughout the working day, all tasks were uploaded onto Listrunner. A Control Centre lead reviewed all uploaded tasks, identified non-medical tasks and either completed these or reassigned them to more appropriate team members. At the end of the pilot, staff provided feedback on their experience via a structured questionnaire.

Results During the pilot, whilst a total of 1080 tasks were uploaded onto Listrunner, $20 \%$ of these were non-medical tasks managed by the Control Centre. The most common tasks managed by Control Centre were chasing specialty reviews (42\%) and chasing investigations (33\%). At the end of the pilot, staff from both wards $(n=19$; Junior Doctors $n=9$, Nurses $n=4$, Consultants $n=2$, Pharmacists $n=2$ and Occupational Therapists $n=2$ ), completed questionnaires. Most doctors $(73 \%)$ found Listrunner easy to use and $56 \%$ of juniors felt that it improved the relevance of their work by reassigning non-medical tasks. Overall, $42 \%$ rated Listrunner as 'useful', whereas $21 \%$ did not find it useful and $56 \%$ felt it improved communication between team members. When asked how Listrunner affected the conduct of ward work, the most popular responses selected were; 'it improved patient flow/discharges' $(n=8)$, 'it speeded up allocation of tasks' $(n=7)$ and 'it prolonged the ward round' $(n=6)$. Whilst $12 / 19(63 \%)$ felt it would be worth adopting Listrunner, 8/19 (42\%) expressed some reservations about using smartphones in front of patients. The main barrier to adopting this technology more widely (according to 58\%) would be the staffing levels and related costs required to replicate the pilot experience.

Conclusions Our data suggest that a large number of tasks currently performed by doctors on acute wards are non-medical tasks. Smartphone technology appears to have potential to improve efficiency and streamline clinical activities, and our early experience may help inform future adoption and further development of this technology.

\section{PTU-140 DOES A DEDICATED INFLAMMATORY BOWEL DISEASE (IBD) PHARMACIST CLINIC IMPROVE PATIENT SAFETY \\ ?}

${ }^{1}$ Alan Steel* ${ }^{2}$ Helen Morgan, ${ }^{2}$ Wei Tan, ${ }^{2}$ Vanessa Marvin, ${ }^{2}$ Mahmood Wahed. ${ }^{1}$ The Royal Liverpool and Broadgreen University Hospitals NHS Trust, Liverpool, UK ${ }^{2}$ Chelsea and Westminster NHS Foundation Trust, London, UK

\subsection{6/gutjnl-2018-BSGAbstracts.518}

Introduction Immunomodulator (IMM) drugs are widely used in the treatment of IBD. These drugs are very effective but also have well recognised, potentially serious, side effects including bone marrow suppression, liver toxicity and pancreatitis. There is a developing role in the UK for pharmacists utilising their specialist knowledge and skills in direct patient facing activities. We aimed to review the impact of the introduction of a specialist IBD pharmacist clinic in the management of IBD patients

Methods IBD patients seen by the IBD pharmacist were identified from our database. Clinical history, demographics, side effects, blood monitoring including TGN levels were recorded. The total number of actual and virtual clinic visits managed by the pharmacist was determined and the outcome of these visits was categorised.

The pharmacist responsibilities included initiation of IMM therapy for patients, medication counselling, prescribing, blood tests and follow up appointments allowing assessment of both clinical response and safety monitoring.

Results Between Nov 2015 and Feb 2017, 367 pharmacist out-patient appointments and 83 pharmacist virtual clinic reviews for 176 IBD patients (Crohn's disease 101, ulcerative colitis 69, IBDU 6) were undertaken. Of the 176 IBD patients, 164 (93\%) were on thiopurines, 9 (5\%) on methotrexate and $3(2 \%)$ on ciclosporin.

Patients visits with the IBD pharmacist were for the following reasons: initiation of IMM treatment (including counselling, dose titration, 2 weekly blood monitoring for the first 2 months), 92 appointments (appts); post initiation, 95 appts; routine 3 monthly monitoring, 145 appts; intensive monitoring (e.g. dose escalation), 45 appts; and dose optimisation (combination therapy with allopurinol) 63 appts. 89\% of clinic appts were managed independently by the pharmacist.

196 appointments resulted in 230 actions in patient management to be undertaken. These were: side effects assessed and pt reassured (37 actions); symptoms assessed and pt reassured (27); adherence support (15); dosing advice (8); dose increased (low thioguanine nucleotide (TGN)) (27), dose decreased (high TGN, abnormal blood tests) (32); allopurinol combination therapy (11); azathioprine switch to 6MP (5); other medication (12); physician review (15); other (34).

As a result of the IBD pharmacist in the clinic, a pharmacy helpline was developed with patients calling or emailing the pharmacist for advice in between clinic visits (122 calls/emails over 37 weeks). 